\title{
A study of corneal thickness, shape and collagen organisation in keratoconus using videokeratography and $\mathrm{X}$-ray scattering techniques
}

\author{
Sally Hayes ${ }^{\mathrm{a}}$, Craig Boote ${ }^{\mathrm{a}}$, Stephen J. Tuft ${ }^{\mathrm{b}}$, Andrew J. Quantock ${ }^{\mathrm{a}}$, Keith M. Meek ${ }^{\mathrm{a}, *}$ \\ ${ }^{a}$ Structural Biophysics Research Group, School of Optometry and Vision Sciences, Cardiff University, Redwood Building, King Edward VII Avenue, \\ Cardiff, CF10 3NB, United Kingdom \\ ${ }^{\mathrm{b}}$ Moorfields Eye Hospital, 162 City Road, London, ECIV 2PD, United Kingdom
}

Received 20 July 2006; accepted in revised form 18 October 2006

Available online 18 December 2006

\begin{abstract}
In keratoconus, the cornea becomes progressively ectactic resulting in severe visual impairment. Here, we use a combination of videokeratography and synchrotron X-ray diffraction to investigate the relationship between corneal shape and thickness, and the distribution and predominant orientation of stromal fibrillar collagen in five keratoconus corneas. In all but the least advanced case, the thinning and ectasia measured in vivo using corneal videokeratography was accompanied by corresponding changes in the relative distribution and orientation of stromal collagen in the excised corneal buttons. Although the most severe case of keratoconus possessed the most pronounced stromal collagen alterations, and only a minor disruption to stromal collagen arrangement was seen in the least advanced case, a variability in the extent of stromal collagen alteration was seen between these clinical extremes. The observed abnormalities in collagen distribution and orientation are consistent with a mechanism of keratoconus progression that involves inter-fibrillar or inter-lamellar slippage causing a redistribution of tissue within the cornea. (c) 2006 Elsevier Ltd. All rights reserved.
\end{abstract}

Keywords: cornea; keratoconus; X-ray scattering; videokeratography

\section{Introduction}

As the cornea is responsible for over two-thirds of the eye's refractive power, any acquired variations in its shape are potentially detrimental to vision. The stroma, which forms the bulk of the cornea, is comprised primarily of water, collagens, proteoglycans and keratocytes. At normal hydration the uniformly small diameter of stromal collagen fibrils and the

\footnotetext{
* Corresponding author. Structural Biophysics Research Group, School of Optometry and Vision Sciences, Cardiff University, Redwood Building, King Edward VII Avenue, POB 905, Cardiff, CF10 3NB, United Kingdom. Tel.: +44 02920 876317; fax: +4402920 874859.

E-mail addresses: denniss@cf.ac.uk (S. Hayes), bootec@cf.ac.uk (C. Boote), s.tuft@ucl.ac.uk (S.J. Tuft), quantockaj@cf.ac.uk (A.J. Quantock), meekkm@cf.ac.uk (K.M. Meek).
}

regular separation distance between them is believed to be regulated by proteoglycans (Borcherding et al., 1975; Chakravarti et al., 1998; Kao and Liu, 2003; Quantock et al., 2001). Collagen fibrils lie parallel to each other within stacked layers (lamellae) which are themselves interspersed with thin, flat keratocytes. Although most lamellae lie parallel to the corneal surface (Komai and Ushiki, 1991), lamellar interweaving is a common feature of the anterior (Radner et al., 1998a) and mid-stroma (Radner and Mallinger, 2002). The specific fibrillar arrangement is believed to provide the cornea with its shape, transparency and strength (Benedek, 1971; Daxer and Fratzl, 1997; Komai and Ushiki, 1991; Maurice, 1957; Meek et al., 2003; Muller et al., 2001; Radner and Mallinger, 2002; Smolek and McCarey, 1990). As a result, abnormalities in the structural organisation of stromal collagen have been implicated in keratoconus, a pathology characterised by 
a progressive thinning and ectasia of the stroma resulting in a cone-shaped cornea and severe irregular astigmatism (Klintworth, 1994).

Despite much research into the pathogenesis of keratoconus, the precise mechanism by which affected corneas progressively thin and steepen remains unknown. In particular, it is unclear if the stromal thinning associated with the disease is caused by an enzyme-mediated loss of collagen (Fini et al., 1992; Kenney et al., 1989, 1994; Kenney and Brown, 2003; Rehany et al., 1982; Sawaguchi et al., 1990, 1994, 1989; Teng, 1963; Zhou et al., 1998), a disruption to fibrillogenesis due to the presence of defective keratocytes (Brookes et al., 2003; Kim et al., 1999; Sherwin et al., 2002; Somodi et al., 1996) or abnormal proteoglycans (Funderburgh et al., 1989), or whether it is due to slippage between lamellae resulting in a redistribution of collagen away from the apex of the cone (Polack, 1976).

In the normal human cornea, more collagen fibrils lie in the superior-inferior and nasal-temporal directions than in any other meridian (Aghamohamadzadeh et al., 2004; Daxer and Fratzl, 1997; Meek et al., 1987). The exact purpose of this arrangement is unknown, but it may be related to the position at which the four extra-ocular rectus muscles insert into the anterior sclera, thereby providing increased tensile strength to prevent corneal distortion during eye movement (Daxer and Fratzl, 1997). Previous studies have shown that, in corneas with keratoconus, the normal orientation of fibrillar collagen is disturbed in the scarred apical region (Daxer and Fratzl, 1997; Radner et al., 1998b) and lamellar interweaving is greatly reduced (Radner et al., 1998b). More recently, it was further shown that the progressive and symmetrical increase of collagen mass from the centre to the edge of the cornea is also disturbed in keratoconus (Meek et al., 2005). These factors likely contribute to the differing biomechanical properties of normal and keratoconus corneas (Andreassen et al., 1980; Nash et al., 1982).

$\mathrm{X}$-ray scattering is a specialised technique that has been used to gain structural information about the constituent collagen in the corneal stroma. The wide-angle equatorial scattering pattern that is produced from the lateral packing of molecules within the stromal collagen fibrils can be used to determine the intermolecular spacing within the fibrils as well as the arrangement and distribution of fibrillar collagen in the intact cornea. Since the collagen molecules are arranged roughly parallel to each other and to the fibril axis, the orientation of the molecules derived from an X-ray scatter image reasonably represents the direction of the collagen fibrils at that position in the cornea. The intensity of X-ray scatter gives quantitative information regarding the relative numbers of fibrils lying in a certain direction, as well as the relative tissue mass at the point through which the beam passes.

Using a combination of videokeratography and X-ray scattering, the current study investigates for the first time the relationship between keratoconus corneal shape and thickness, and abnormalities in stromal collagen arrangement.

\section{Materials and methods}

\subsection{Tissue}

Five keratoconus corneal buttons, of 7.5, 8 or $8.5 \mathrm{~mm}$ diameter (Table 1), were obtained at the time of penetrating keratoplasty in accordance with the tenets of the Declaration of Helsinki. A pre-operative central keratometric reading was used to classify the severity of the keratoconus (Buxton et al., 1984). In each case the indication for surgery was the inability of the patient to achieve good vision with contact lenses.

The individual buttons were marked at the 12 o'clock position with a surgical skin marker pen. The samples were then wrapped in polyvinylidene chloride catering film (Clingfilm $^{\mathrm{TM}}$, Superdrug Stores Plc., Croydon, UK) to limit dehydration and frozen at $-80{ }^{\circ} \mathrm{C}$. This is an acceptable means of storing corneal tissue prior to examination by synchrotron X-ray scattering (Daxer and Fratzl, 1997; Fratzl and Daxer, 1993; Fullwood and Meek, 1994).

Table 1

Sample details of normal and keratoconus corneas

\begin{tabular}{lll}
\hline Sample & Details & Classification $^{\mathrm{a}}$ \\
\hline $\mathrm{N} 1$ & $\begin{array}{l}\text { Normal cornea with scleral rim. } \\
\text { Right eye, 77-year-old male. }\end{array}$ & Normal \\
N2 & $\begin{array}{l}\text { Normal cornea with scleral rim. } \\
\text { Left eye, 77-year-old male. }\end{array}$ & Normal \\
N3 & $\begin{array}{l}\text { Normal cornea with scleral rim. } \\
\text { Left eye, 84-year-old female. }\end{array}$ & Normal \\
N4 & $\begin{array}{l}\text { Normal cornea with scleral rim. } \\
\text { Right eye, 77-year-old, male. }\end{array}$ & Normal \\
K1 & $\begin{array}{l}\text { Keratoconus button (7.5 mm). Right } \\
\text { eye, 32-year-old female. Central } \\
\text { cone with apical corneal scar. } \\
\text { Pre-op BCVA }\end{array}$ & Advanced (54.3D) \\
& contact lens was 6/12. & \\
& Keleral &
\end{tabular}

K2 Keratoconus button $(7.5 \mathrm{~mm})$. Right eye, 37-year-old male. Central cone

Advanced (58.3D) with superficial apical scars. Pre-op BCVA ${ }^{\mathrm{b}}$ with corneal contact lenses was 6/18.

K3 Keratoconus button (8 mm). Right eye, 32-year-old male.

Central cone with a superficial axial scar. Pre-op BCVA ${ }^{\mathrm{b}}$ with rigid gas permeable contact lenses was $6 / 18$.

K4 Keratoconus button (8 mm). Right eye, 32-year-old male. Inferior displaced cone with a superficial scar above the visual axis. Pre-op $\mathrm{BCVA}^{\mathrm{b}}$ with a corneal contact lenses was $6 / 12$. Keratoconus button $(8.5 \mathrm{~mm})$. Right eye, 39-year-old male. Large, steep central cone with minimal corneal scarring. Pre-op BCVA ${ }^{\mathrm{b}}$ with a scleral contact lens was 6/18.

\footnotetext{
${ }^{a}$ Classification is based on the central keratometric reading (shown in brackets).

${ }^{\mathrm{b}}$ Pre-operative best corrected visual acuity.
} 
The normal right and left corneas with scleral rim (from the same donor) were provided by Manchester Eye Hospital, UK. They were obtained within $3 \mathrm{~h}$ of death and a suture was inserted in the superior aspect of the sclera to mark the orientation. Two other normal corneas (N3 and N4) of known orientation were obtained from the Bristol Eye Bank, UK (Table 1). The samples were wrapped in polyvinylidene chloride catering film, frozen in liquid nitrogen cooled isopentane and stored at $-80{ }^{\circ} \mathrm{C}$.

\subsection{Videokeratography}

Prior to surgery, a videokeratography image of all five keratoconus corneas was recorded using the Orbscan II $^{\mathrm{TM}}$ imaging system (Bausch and Lomb, London, UK) to produce topography maps of anterior surface elevation and corneal thickness. The regions of greatest anterior surface elevation ( $>0.045 \mathrm{~mm}$ above a 'best fit' reference sphere) and maximal tissue thinning (defined as a pachymetry reading of $<450 \mu \mathrm{m})$ are highlighted by a black solid line and a blue broken line, respectively, in Fig. 1. For the purposes of this study the combined regions of greatest anterior surface elevation and maximal tissue thinning will be referred to as the 'apical region'.

\subsection{X-ray scattering - data collection}

All X-ray scattering data were collected on Station 14.1 at the UK Synchrotron Radiation Source (Daresbury Laboratory, Warrington, UK), using an X-ray beam focused to $200 \times$ $200 \mu \mathrm{m}$ at the specimen with a wavelength of $0.1488 \mathrm{~nm}$. The samples were wrapped tightly in polyvinylidene chloride catering film to prevent tissue dehydration during data collection and placed in their correct orientation in a sealed sample holder enclosed between two sheets of Mylar (DuPont Teijin Films ${ }^{\mathrm{TM}}$, London, UK). The sample holder was then carefully secured onto a computer-operated translation stage (Newport Spectra-Physics Ltd., Newbury, UK) with the anterior surface of the cornea facing the X-ray beam. A series of wide-angle Xray scattering images resulting from a beam exposure of between 45 and $75 \mathrm{~s}$ were collected over the entire sample at regular intervals of $0.4-0.5 \mathrm{~mm}$ in the case of the normal corneas, and $0.25 \mathrm{~mm}$ for each of the keratoconus buttons. The exposure time remained constant for individual samples but varied between samples due to differences in the beam intensity at the time of data collection. The resulting X-ray scattering images were recorded on a Quantum 4R CCD detector (ADSC, Poway, CA) placed $15 \mathrm{~cm}$ behind the cornea. The X-ray scattering patterns were calibrated using the $0.304 \mathrm{~nm}$ X-ray reflection from powdered calcite.

\subsection{X-ray scattering - data analysis}

The angular distribution of scatter intensity around the collagen intermolecular reflection (Fig. 2A) was measured to form a $0-360^{\circ}$ distribution pattern using Optimus 6.5
(Media Cybernetics, Wokingham, UK) image analysis software and Excel (Microsoft, UK) spreadsheets (Fig. 2B). Further details of this analysis procedure are provided by Newton and Meek (1998). To take into account the variations in beam intensity and exposure time between samples, the intensity profile for each image was normalised against the average X-ray intensity at the time of data collection (recorded by an ion chamber) multiplied by the beam exposure time. The distance from the centre of the scattering pattern to the intermolecular reflection was measured and calibrated against the known spacing of calcite to obtain the intermolecular Bragg spacing of collagen. Based on the assumption that collagen molecules are packed in a pseudo-hexagonal lattice, the Bragg spacing was multiplied by a factor of 1.11 to give the mean intermolecular centre-to-centre spacing (Klug and Alexander, 1974).

The total area under the intensity profile (i.e. the total X-ray scatter) is proportional to the total amount of fibrillar collagen in the path of the X-ray beam - the more collagen, the greater the intensity of X-ray scatter (Newton and Meek, 1998). The total X-ray scatter is comprised of two components: the scatter arising from collagen fibrils that lie equally in all directions within the plane of the cornea (isotropic scatter) and the scatter from collagen fibrils that adopt a preferred orientation (aligned scatter) (Fig. 2B). Removal of the isotropic scatter leaves only the scatter from preferentially aligned lamellae, which will be referred to throughout as 'aligned collagen' (Fig. 2C).

The area under each intensity profile (Fig. 2B, C) was summed to produce a numeric value of scatter intensity for both total collagen and aligned collagen. The ratio of the scatter intensity from aligned collagen as a proportion of the total collagen scatter intensity was defined as the "index of orientation'. In this scheme a value of 1 indicates that all of the collagen present is preferentially aligned and a value of 0 means that the collagen is distributed equally in all directions within the plane of the cornea. By plotting each calculated intensity value/index on a grid relating to corneal position, contour maps showing the distribution of total collagen scatter (and hence relative fibrillar mass) and the indices of orientation were produced. By presenting the distribution of aligned collagen as an index of orientation, the reduced stromal thickness in keratoconus corneas is taken into account and so the distribution of aligned collagen can be directly compared between normal and keratoconus corneas.

In order to show the preferred orientation of aligned collagen within a sample, each profile of aligned collagen scatter was shifted by $90^{\circ}$ to account for the fact that $\mathrm{X}$ rays are scattered at right angles to the orientation of the collagen molecules. The resulting scatter intensity profile was then transformed into polar co-ordinates to produce vector plots (Fig. 2D) (Connon and Meek, 2003). The distance from the origin of a vector plot to the edge, at any given angle, represents the intensity of scatter from aligned collagen lying in a particular direction. The individual vector plots were compiled onto a grid relating to corneal position to form a 'vector plot map', showing the preferred orientation of aligned collagen over the entire 


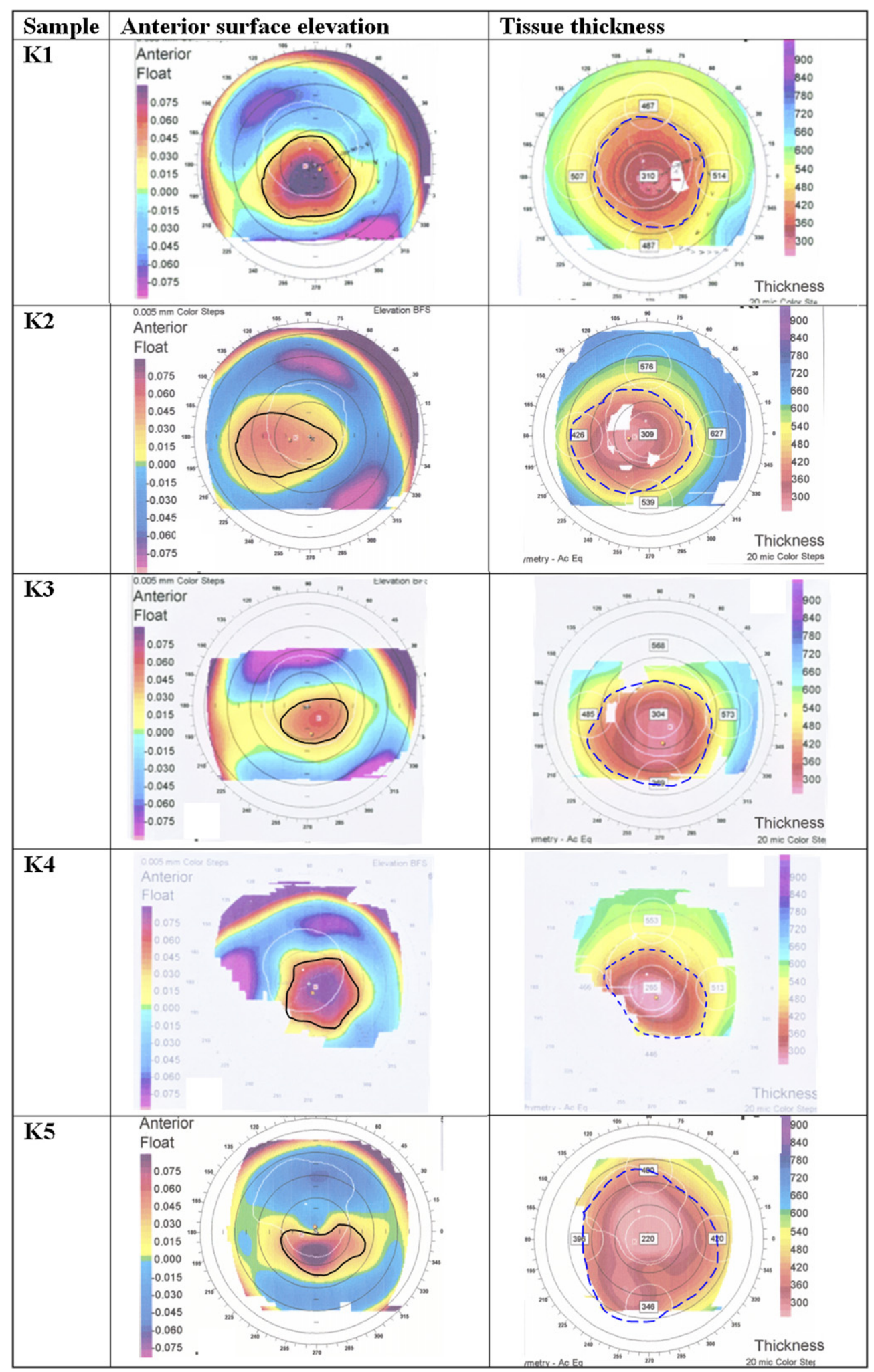

Fig. 1. Orbscan videokeratography of five keratoconus corneas (K1-K5) showing their anterior surface elevation and pachymetry. The regions of greatest surface elevation $(>0.045 \mathrm{~mm}$ above a 'best fit' reference sphere) and minimal tissue thickness $(<450 \mu \mathrm{m})$ have been highlighted by a black solid line and blue broken line, respectively.

sample. Due to variations in the amount of aligned collagen across each sample the vector plots were scaled down by factors of $3,2,1.5$ and 1 and coloured black, brown, red and orange, respectively, in Figs. 6 and 7.
As normal left and right corneas are enantiomorphic in terms of the mass distribution of aligned collagen (Boote et al., 2006), the right keratoconus corneal buttons used in this study are compared to the central $8 \mathrm{~mm}$ region of a normal 


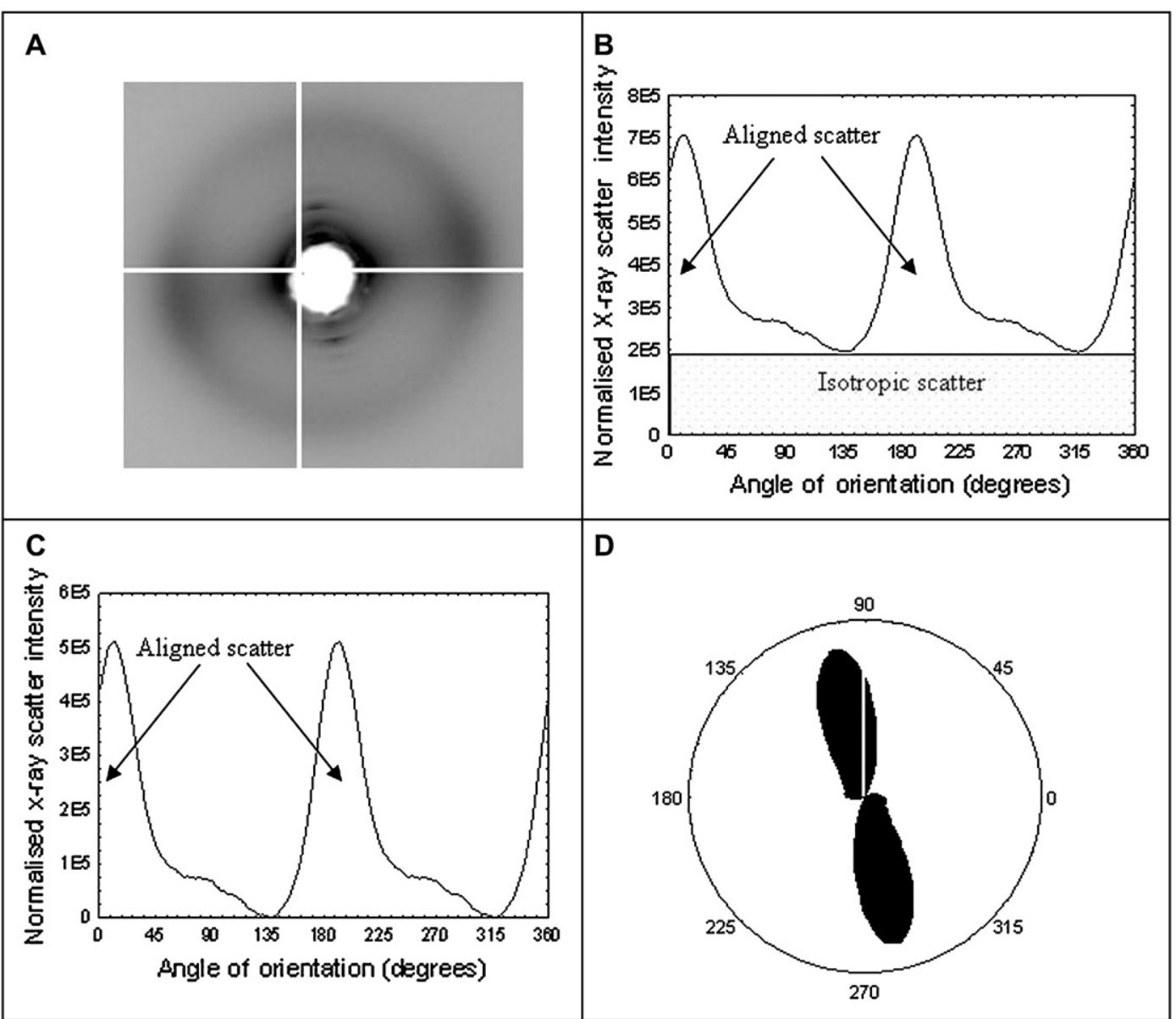

Fig. 2. X-ray scatter data analysis. (A) An example of a wide-angle X-ray scattering pattern taken by passing a beam of X-rays through the cornea parallel to the optical axis. Intensity profiles of total collagen X-ray scatter (B) and preferentially aligned collagen alone (C) are shown as a function of angular position around the scatter pattern (A). The intensity profile of scatter from the preferentially aligned collagen is shifted by $90^{\circ}$ to account for the fact that X-rays are scattered at right angles to the fibril axis. The resulting profile is then converted into a vector plot (D), in which the radial extent in any given direction represents the amount of collagen preferentially orientated in that direction.

right cornea (N1). X-ray scattering measurements of intermolecular spacing are, however, presented for all four normal corneas examined here.

\section{Results}

\subsection{Intermolecular spacing of collagen in normal and keratoconus corneas}

Measurements of intermolecular spacing made at 0.4$0.5 \mathrm{~mm}$ intervals along the nasal-temporal meridian of the central $8 \mathrm{~mm}$ of four normal corneas revealed a striking uniformity across individual corneas. When measured at an even higher resolution of $0.25 \mathrm{~mm}$, the same consistency in intermolecular spacing was observed across the entire horizontal meridian of all keratoconus buttons. Importantly no variation in intermolecular spacing occurred between the apical and para-apical regions in any of the keratoconus corneas and a statistical analysis of variance (ANOVA) revealed that the average intermolecular spacing did not differ significantly between normal $(1.77 \pm 0.008 \mathrm{~nm})$ and keratoconus corneas $(1.77 \pm 0.007 \mathrm{~nm})$.

\subsection{Collagen distribution in normal and keratoconus corneas}

Contour plots showing the relative distribution of total collagen in one normal and five keratoconus corneal buttons are shown in Fig. 3. Using the same colour scale thresholds for each specimen it is evident that the distribution of total collagen in the normal cornea (N1) is uniform across the central $8 \mathrm{~mm}$ region, in line with the previous findings (Aghamohamadzadeh et al., 2004; Boote et al., 2006).

On the other hand, all keratoconus buttons showed a nonuniform distribution of collagen scatter. In all cases the highlighted region of reduced corneal thickness coincides with a region - or in some cases (K3 and K5) multiple regions - of abnormally low collagen scatter intensity. Within the apical region, patches of increased collagen scatter were often seen in close proximity to the areas of reduced collagen scatter, with the least advanced case showing the least collagen loss (K1) and the 


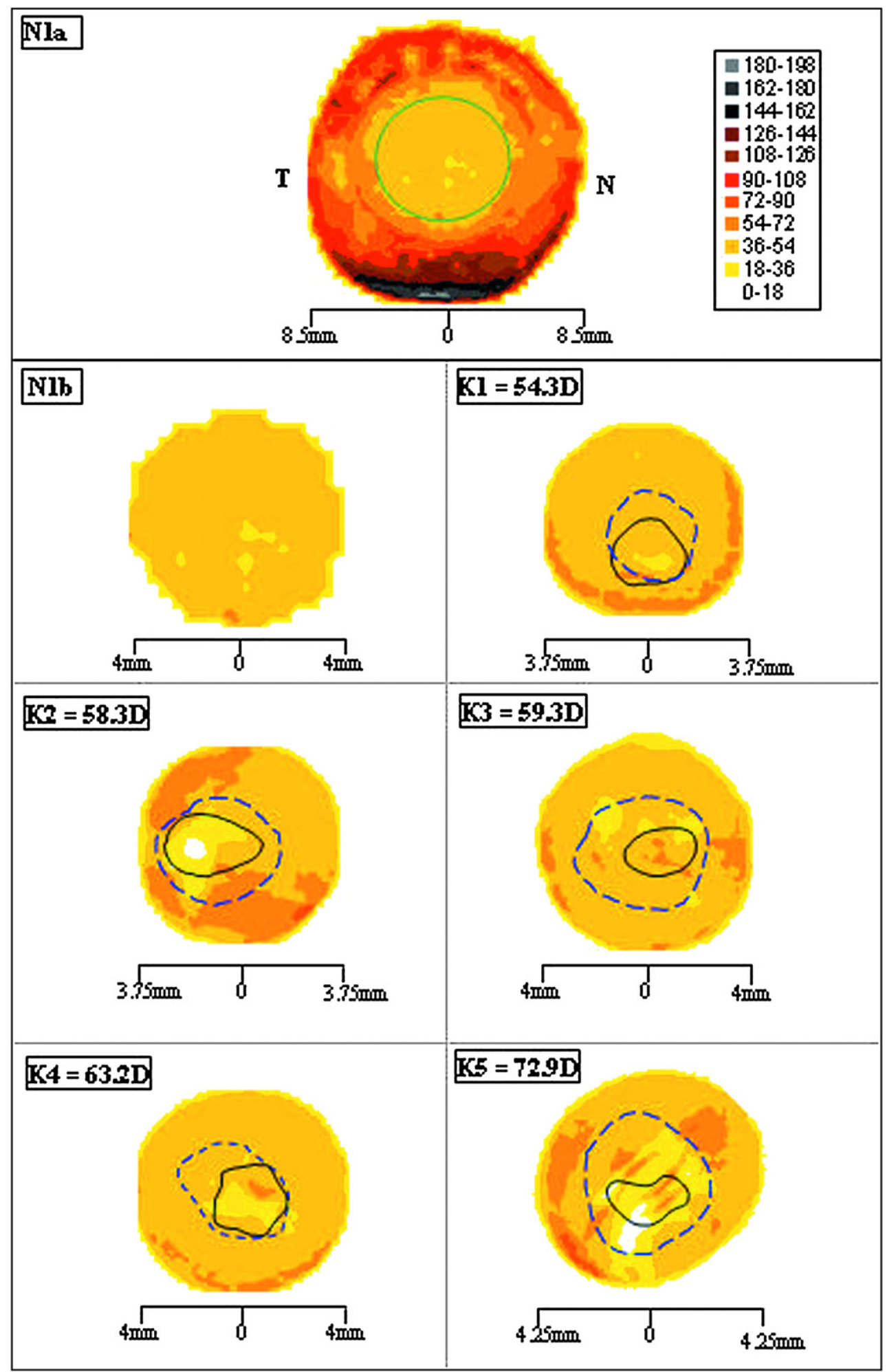

Fig. 3. Total collagen distribution. Contour maps showing the relative distribution of total collagen in a normal cornea with scleral rim (N1a) and in the central $7.5-8.5 \mathrm{~mm}$ of the same cornea and five keratoconus corneas (N1b, K1-K5). Green line in N1a denotes central 8 mm region - shown enlarged as N1b. Regions of greatest anterior surface elevation (black solid line) and tissue thinning (blue broken line) are highlighted.

most severe keratoconus (K5) associated with the most extensive loss. There was, however, variability in collagen distribution between these clinical extremes, so it is likely that a severe phenotype must be reached before the link between disease severity and collagen mass redistribution is manifest.

\subsection{Preferentially aligned collagen distribution in normal and keratoconus corneas}

Fig. 4 shows the distribution of aligned collagen scatter in the normal and keratoconus corneas depicted in Fig. 3. In the 


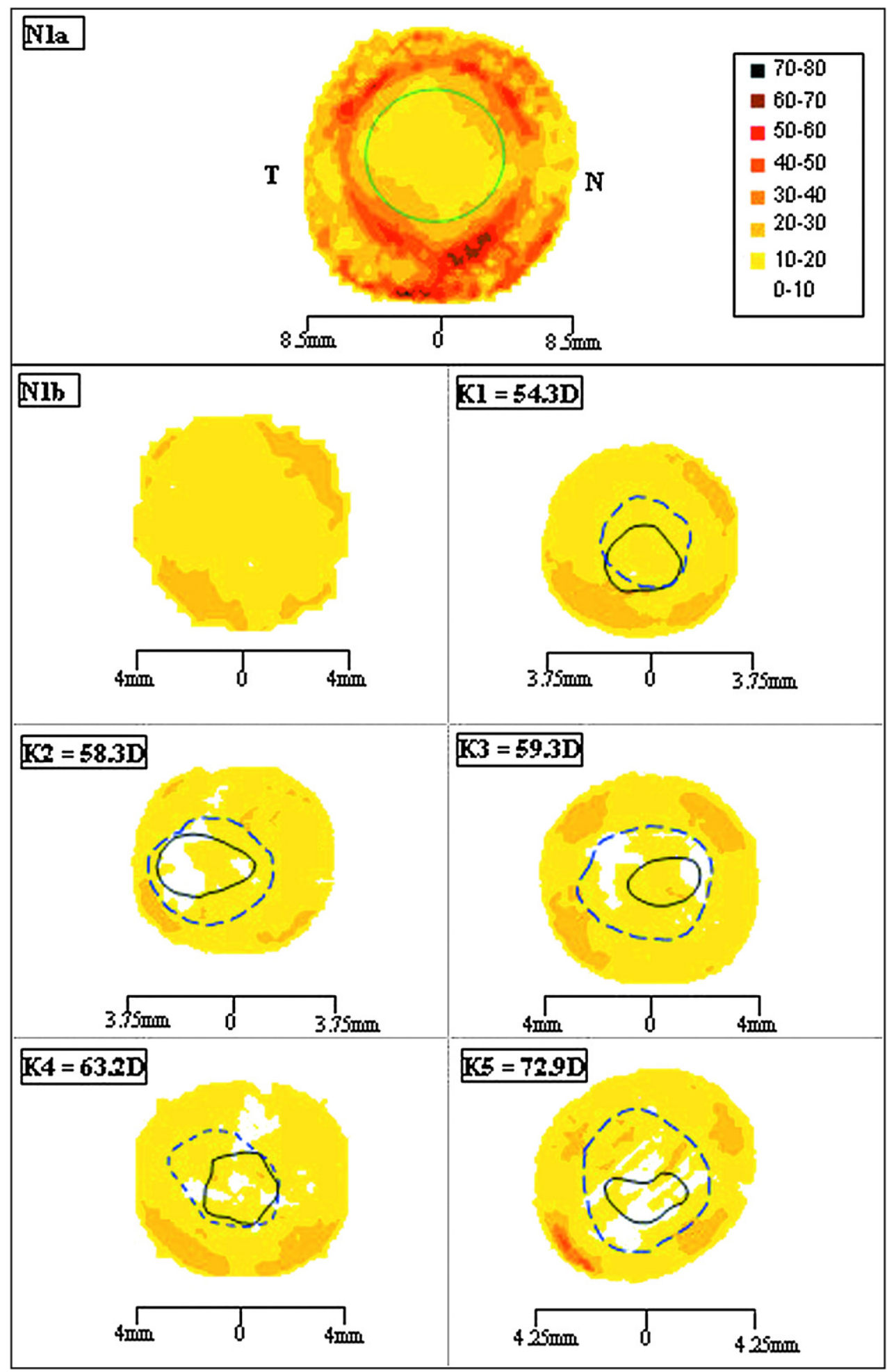

Fig. 4. Preferentially aligned collagen distribution. Distribution of aligned collagen in a normal cornea with scleral rim (N1a) and in the central 7.5-8.5 mm of the same cornea and five keratoconus corneas (N1b, K1-K5). Green line in N1a denotes central $8 \mathrm{~mm}$ region - shown enlarged as N1b. Regions of greatest anterior surface elevation (black solid line) and tissue thinning (blue broken line) are highlighted.

normal cornea (N1), the amount of preferentially aligned collagen remains constant across the central $5 \mathrm{~mm}$ of the cornea but increases in each of the four quadrants to form a skewed diamond shaped arrangement. This arrangement is characteristic of the normal human cornea (Aghamohamadzadeh et al.,
2004; Boote et al., 2006). The normal diamond shape seen in the distribution of aligned collagen was disturbed to varying extents in all keratoconus corneal buttons. The least advanced case (K1) showed only a small change from normal (N1b) and in $\mathrm{K} 4$, two sides of the diamond were disturbed. In the 


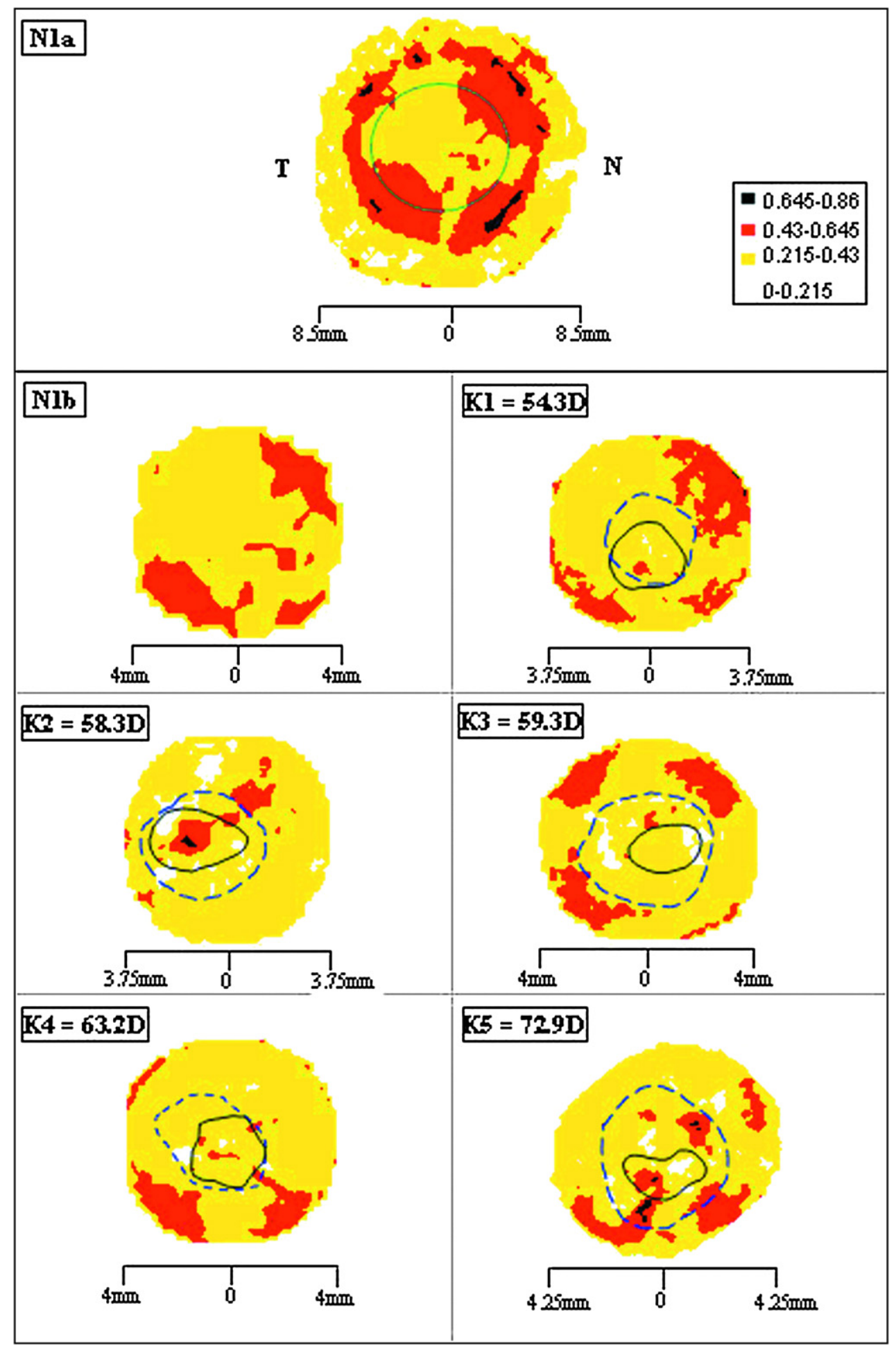

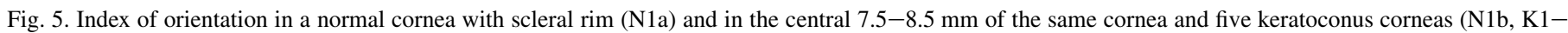

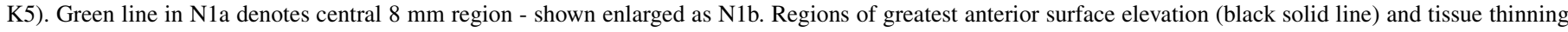
(blue broken line) are highlighted.

remaining three keratoconus buttons (K2, K3 and K5), all four quadrants showed abnormalities in the distribution of aligned collagen scatter.

With the exception of K1, a reduction in aligned collagen scatter was apparent in the apical region of each keratoconus cornea. Although a general trend indicated a relationship between the severity of the disease and the reduction in aligned collagen scatter (with the least advanced keratoconus showing no change in aligned collagen scatter in the central cornea and the most severe case (K5) displaying a large region of reduced aligned collagen scatter), marked variations were observed between specimens. For example, in keratoconus 
cornea $\mathrm{K} 4$ the region of reduced aligned collagen scatter extended beyond the apical region, and in another cornea (K3) two distinct regions of reduced aligned collagen scatter were seen close to the edge of the apical region.

When the distribution of aligned collagen was plotted as the index of orientation (Fig. 5), it was found that in the normal cornea (N1a) the index of orientation increased in all four quadrants of its periphery, with the greatest increase occurring in the superior-nasal and inferior-temporal quadrants. Within the central $8 \mathrm{~mm}$ region, this increase in the index of orientation can still be clearly seen in the superior-nasal and inferior-temporal quadrants of the normal cornea (N1b). In three out of five of the keratoconus buttons examined $(\mathrm{K} 2, \mathrm{~K} 4$ and $\mathrm{K} 5)$ the index of orientation failed to increase in one or more of the quadrants, and an abnormal increase in the index of orientation was observed close to the apex of the cone. In all but the least advanced case of keratoconus, patches of abnormally low index of orientation (visible as white areas in Fig. 5) were observed both inside and outside the apical region.

\subsection{Collagen orientation in normal and keratoconus corneas}

In the central $6 \mathrm{~mm}$ of the normal cornea $(\mathrm{N} 1)$ the preferred alignment of collagen fibrils is in the superior-inferior and nasal-temporal meridians (Fig. 6). This gives rise to the characteristic cross-shaped vector plots seen in previous X-ray scattering studies of normal human corneas (Aghamohamadzadeh et al., 2004; Boote et al., 2006). Beyond the central $6 \mathrm{~mm}$ region, the preferred orthogonal orientation is gradually

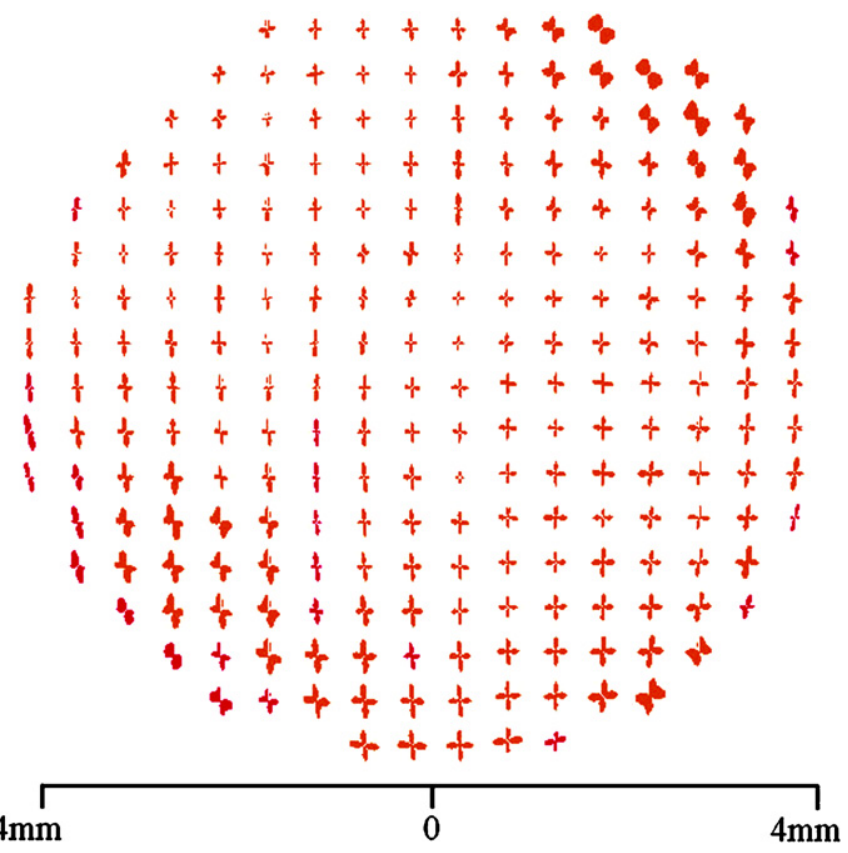

Fig. 6. Preferred fibril orientation in the normal cornea. Preferred fibril direction in the central $8 \mathrm{~mm}$ of a normal cornea (N1). Due to variations in the amount of aligned collagen across the cornea it was necessary to scale down the larger vector plots by a factor of 1.5 (red). obscured by the presence of additional collagen aligned tangentially to the edge of the cornea.

The normal preferred orientation of collagen was disturbed to a variable extent in each of the keratoconus buttons but in all cases the extent of disruption decreased with distance from the apex (Fig. 7). In the least advanced case (K1) the preferred orientation of collagen was normal throughout most of the $7.5 \mathrm{~mm}$ button. In contrast, the most severe case (K5) showed an abnormal orientation of collagen throughout the entire $8.5 \mathrm{~mm}$ button region. The remaining corneas (K2-K4) all exhibited extreme changes in collagen orientation that extended well beyond the apical region, however, a precise relationship between disease severity (in terms of dioptric power) and the extent of the structural alterations was not apparent.

\section{Discussion}

In this study, a combined approach of videokeratography and X-ray scattering was used to investigate the link between changes in corneal shape and thickness, and alterations in stromal ultrastructure in keratoconus, to improve understanding of the mechanism by which ectasia occurs and progresses.

$\mathrm{X}$-ray scattering is a unique method to measure the lateral spacing between individual fibril-forming collagen molecules to less than $1 \mathrm{~nm}$ resolution. This spacing is influenced by both the hydration of the fibrils (Fratzl and Daxer, 1993; Meek et al., 1991) and by the degree of molecular cross-linking (Malik et al., 1992). Any changes in this spacing within different parts of the keratoconus cornea could cause alterations in the regional biomechanical properties of the tissue. We have demonstrated here, however, that within any given keratoconus cornea, even the most severe case, there were no regional differences in this spacing, indicating that the collagen arrangement within fibrils does not vary between the apex and the less affected peripheral region. Fullwood et al. (1992) showed that at the optical centre of the keratoconus cornea there is a small decrease in the intermolecular spacing compared to the normal cornea. We were unable to confirm this result. It is worth noting that the proportional relationship assumed to exist between the intensity of X-ray scatter and the amount of fibrillar collagen in the path of the beam is based on the assumption that the hydration of the collagen fibrils does not vary across the cornea. In this study, the consistency in intermolecular spacing across the central $8 \mathrm{~mm}$ region of normal and keratoconus corneas supports this assumption. It also suggests that there are no detectable regional variations in collagen cross-linking that may cause some parts of the keratoconic cornea to be weaker than others.

We have shown that the corneal ectasia and thinning associated with keratoconus are accompanied by changes in the relative distribution and orientation of collagen within the corneal stroma. Although such stromal abnormalities in keratoconus corneas have previously been shown by X-ray scattering and scanning electron microscopy (Daxer and Fratzl, 1997; Meek et al., 2005; Radner et al., 1998b; 


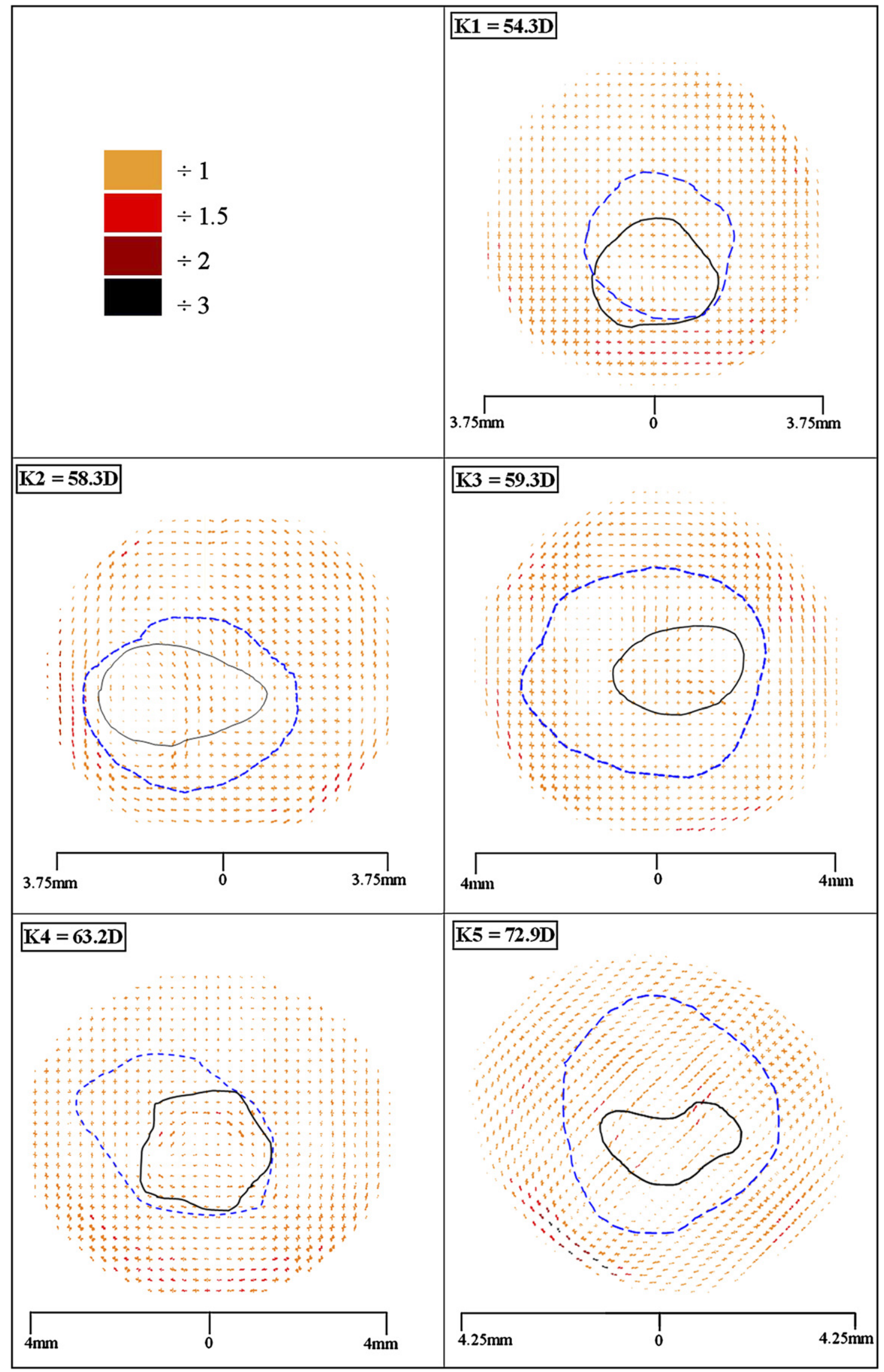

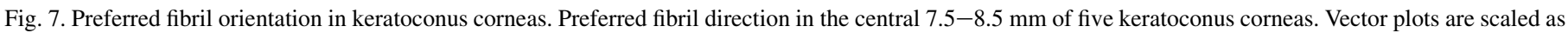
indicated in the key. Regions of greatest anterior surface elevation (black solid line) and tissue thinning (blue broken line) are highlighted. 
Sawaguchi et al., 1998) this study goes further, to examine for the first time the relationship between the altered arrangement and distribution of collagen and the specific shape and thickness of the diseased cornea. In normal cornea the total collagen scatter is essentially constant across the central $8 \mathrm{~mm}$, while, in keratoconus, an uneven distribution of collagen is invariably found. As would be expected, the region of greatest tissue thinning coincided with a decrease in total collagen scatter. However, the present study shows that in some cases the structural changes are far more complex than the videokeratography might suggest. For example, in one keratoconus button, the videokeratography showed a large region of reduced tissue thickness, whereas X-ray scattering data revealed that this region was actually comprised of two distinct regions of stromal thinning (K3 in Figs. 3 and 4). The pre-set step size assigned to the colour scale of the Orbscan topography system may be responsible for its failure to detect such subtle local changes in pachymetry.

Previously it was found that stromal thinning in keratoconus was not accompanied by a compaction of collagen fibrils (Fullwood et al., 1992), as is the case in macular corneal dystrophy, another corneal disease in which the corneal stroma thins (Quantock et al., 1990). It was reasoned, therefore, that stromal thinning in keratoconus is most likely due to a loss of fibrils from the central cornea. This is verified by the current findings. However, the plots in Fig. 3 also imply that some parts of the keratoconus buttons show increased scatter. The $\mathrm{X}$-ray maps are two-dimensional projections of the curved cornea and, as such, increased corneal steepness will lead to an increase in the fibrillar mass sampled. Our previous calculations (Meek et al., 2005) suggest that this effect is small, so the possibility cannot be excluded that regions of increased scatter are those to which mass has been redistributed, perhaps by a process of lamella slippage (Meek et al., 2005; Polack, 1976). Unfortunately, it is not easy to correlate these regions with Orbscan thickness data for the reasons outlined above.

When the distribution of aligned lamellae is presented as an index of orientation (Fig. 5), normal corneas show highly reproducible increases in the orientation in three quadrants superior-nasal, inferior-temporal and inferior-nasal (Boote et al., 2006). The least advanced cornea (K1) showed the same pattern, but the other keratoconus buttons did not. A change in the local index of orientation may be explained either by a preferential loss of isotropic or aligned collagen during stromal thinning or by a redistribution of collagen mass. The latter is more likely since abnormalities in the distribution of collagen are also accompanied by changes in the preferred orientation of lamellae (Fig. 7).

It has been shown in this paper and elsewhere (Aghamohamadzadeh et al., 2004; Boote et al., 2006) that the increase in aligned collagen mass in the periphery of the normal cornea forms a highly reproducible skewed diamond shaped arrangement. This specific arrangement is thought to be due to the presence of anchoring fibrils that traverse the peripheral cornea and contribute to the flattening of the cornea inside the limbus (Aghamohamadzadeh et al., 2004). Abnormalities in the distribution of aligned collagen were observed in the peripheral region of all keratoconus corneas with the possible exception of the least advanced (K1) (Fig. 4), although the extent of the disruption appeared to be independent of the disease severity and the size and shape of the cone. The absence of the skewed diamond shape arrangement of aligned collagen in keratoconus suggests that the anchoring fibrils are not present or that they occur at a distance beyond the central $8 \mathrm{~mm}$ region under investigation.

We have shown that in less advanced keratoconus, corneal ectasia can be present with little loss of tissue mass and only minor alterations in collagen lamellar orientation. As the disease progresses, there is a variability between keratoconus corneas in terms of the relationship between the severity of the disease and the extent of the structural alterations. Irrespective of the initial pathogenesis, which probably involves an up-regulation of degradative enzymes (Kenney et al., 1994; Kenney and Brown, 2003; Sawaguchi et al., 1989; Zhou et al., 1998) leading to some loss of collagen (Yue et al., 1984), the findings presented in this paper support a mechanism of tissue thinning in keratoconus corneas that also involves lamellar or fibrillar slippage. This idea is supported by the observation of abnormal collagen-proteoglycan arrangement in keratoconus corneas (Fullwood et al., 1990, 1992). It is also supported by the observation of less lamellar interweaving, a factor which may influence the biomechanical properties of the tissue and facilitate such a mechanism of stromal thinning (Radner et al., 1998b). As a consequence we propose that the development of interventional cross-linking strategies that not only protect the collagen from degradation but that may also limit collagen fibrillar slippage (Wollensak et al., 2003), should be beneficial to delay or retard the progression of keratoconus.

\section{Acknowledgements}

The authors wish to thank Dr. Mike MacDonald and the staff at the UK Synchrotron X-ray Source (Daresbury, UK) for help with data collection. We also thank Tom Kelly (Manchester Eye Hospital, UK) and Val Smith (UK Corneal Transplant Service Eye Bank, Bristol, UK) for provision of specimens. This study received programme grant support from the Medical Research Council (Grant G0001033) and the Council for the Central Laboratory of Research Councils (Grant 18).

\section{References}

Aghamohamadzadeh, H., Newton, R.H., Meek, K.M., 2004. X-ray scattering used to map the preferred collagen orientation in the human cornea and limbus. Structure 12, 249-256.

Andreassen, T., Simonsen, A., Oxlund, H., 1980. Biomechanical properties of keratoconus and normal cornea. Experimental Eye Research 31, 435-441.

Benedek, G.B., 1971. Theory of transparency of the eye. Applied Optics 10, 459-472.

Boote, C., Hayes, S., Abahussin, M., Meek, K., 2006. Mapping collagen organisation in the human cornea: left and right eyes are structurally distinct. Investigative Ophthalmology and Visual Science 47, 901-908.

Borcherding, M.S., Balccik, L.J., Sittig, R.A., Bizzel, J.W., Breen, M., Weinstein, H.G., 1975. Proteoglycans and collagen fibre organisation in human corneoscleral tissue. Experimental Eye Research 21, 59-70. 
Brookes, N.H., Loh, I.P., Clover, G.M., Poole, C.A., Sherwin, T., 2003. Involvement of corneal nerves in the progression of keratoconus. Experimental Eye Research 77, 515-524.

Buxton, J.N., Keates, R.H., Hoefle, F.B., 1984. The contact lens correction of keratoconus. In: Dabezies, O.H.J. (Ed.), Contact lenses: The CLAO Guide to Basic Science and Clinical Practice. Grune and Stratton, Orlando, p. 55.5.

Chakravarti, S., Magnuson, T., Lass, J.H., Jespen, K.L., LaMantia, C., Carroll, H., 1998. Lumican regulates collagen fibril assembly: skin fragility and corneal opacity in the absence of lumican. The Journal of Cell Biology 141, 1277-1286.

Connon, C.J., Meek, K., 2003. Organization of corneal collagen fibrils during the healing of trephined wounds in rabbits. Wound Repair and Regeneration $11,71-78$.

Daxer, A., Fratzl, P., 1997. Collagen fibril orientation in the human corneal stroma and its implications in keratoconus. Investigative Ophthalmology and Visual Science 38, 121-129.

Fini, M.E., Yue, B.Y., Sugar, J., 1992. Collagenolytic/gelatinolytic metalloproteinases in normal and keratoconus corneas. Current Eye Research 11, 849-862.

Fratzl, P., Daxer, A., 1993. Structural transformation of collagen fibrils in the corneal stroma during drying: an X-ray scattering study. Biophysical Journal 64, 1210-1214.

Fullwood, N.J., Meek, K.M., 1994. An ultrastructural, time-resolved study of freezing in the corneal stroma. Journal of Molecular Biology 236, $749-758$

Fullwood, N.J., Meek, K.M., Malik, N.S., Tuft, S.J., 1990. A comparison of proteoglycan arrangement in normal and keratoconus human corneas. Biochemical Society Transactions 18, 961-962.

Fullwood, N.J., Tuft, S.J., Malik, N.S., Meek, K.M., Ridgway, A.E.A., Harrison, R.J., 1992. Synchrotron X-ray diffraction studies of keratoconus corneal stroma. Investigative Ophthalmology and Visual Science 33, 1734-1741.

Funderburgh, J.L., Panjwani, N., Conrad, G.W., Baum, J., 1989. Altered keratan sulphate epitopes in keratoconus. Investigative Ophthalmology and Visual Science 30, 2278-2281.

Kao, W.W.Y., Liu, C.Y., 2003. Roles of lumican and keratocan on corneal transparency. Glycoconjugate Journal 19, 275-285.

Kenney, M., Chwa, M., Escobar, M., Brown, D., 1989. Altered gelatinolytic activity by keratoconus corneal cells. Biochemical and Biophysical Research Communications 161, 353-357.

Kenney, M., Chwa, M., Opbroek, A.J., Brown, D.J., 1994. Increased gelatinolytic activity in keratoconus cultures. A correlation to an altered matrix metalloproteinase-2/tissue inhibitor of metalloproteinase ratio. Cornea 13, 114-124.

Kenney, M.C., Brown, D.J., 2003. The cascade hypothesis of keratoconus. Contact Lens and Anterior Eye 26, 139-146.

Kim, W.J., Rabinowitz, Y.S., Meisler, D.M., Wilson, S.E., 1999. Keratocyte apoptosis associated with keratoconus. Experimental Eye Research 69, 475-481.

Klintworth, G.K., 1994. Degenerations, depositions and miscellaneous reactions of the ocular anterior segment. In: Klintworth, G.K., Garner, A. (Eds.), Pathobiology of Ocular Disease: A Dynamic Approach, second ed. Marcel Dekker, New York.

Klug, H., Alexander, L.E., 1974. X-ray Diffraction Procedures for Crystalline and Amorphous Materials. New York.

Komai, Y., Ushiki, T., 1991. The three-dimensional organisation of collagen fibrils in the human cornea and sclera. Investigative Ophthalmology and Visual Science 32, 2244-2258.

Malik, N.S., Moss, S.J., Ahmed, N., Furth, A.J., Wall, R.S., Meek, K.M., 1992. Aging of the human corneal stroma - structural and biochemical changes. Biochimica Et Biophysica Acta 1138, 222-228.

Maurice, D.M., 1957. The structure and transparency of the cornea. Journal of Physiology 136, 263-286.

Meek, K., Blamires, T., Elliot, G., Gyi, T.J., Nave, C., 1987. The organisation of collagen fibrils in the human corneal stroma: a synchrotron X-ray diffraction study. Current Eye Research 6, 841-846.

Meek, K.M., Fullwood, N.J., Cooke, P.H., Elliott, G.F., Maurice, D.M., Quantock, A.J., Wall, R.S., Worthington, C.R., 1991. Synchrotron X-ray- diffraction studies of the cornea, with implications for stromal hydration. Biophysical Journal 60, 467-474.

Meek, K.M., Leonard, D.W., Connon, C.J., Dennis, S., Khan, S., 2003. Transparency, swelling and scarring in the corneal stroma. Eye 17, 927-936.

Meek, K.M., Tuft, S.J., Huang, Y., Gill, P.S., Hayes, S., Newton, R.H., Bron, A.J., 2005. Changes in collagen orientation and distribution in keratoconus corneas. Investigative Ophthalmology and Visual Science 46, 1948-1956.

Muller, L.J., Pels, E., Vrensen, G.F.J.M., 2001. The specific architecture of the anterior stroma accounts for maintenance of corneal curvature. British Journal of Ophthalmology 85, 437-443.

Nash, I., Greene, P., Foster, S., 1982. Comparison of mechanical properties of keratoconus and normal cornea. Experimental Eye Research 35, 413-423.

Newton, R.H., Meek, K.M., 1998. Circumcorneal annulus of collagen fibrils in the human limbus. Investigative Ophthalmology and Visual Science 39, 1125-1134.

Polack, F.M., 1976. Contributions of electron microscopy to the study of corneal pathology. Survey of Ophthalmology 20, 375-414.

Quantock, A.J., Meek, K.M., Chakravarti, S., 2001. An X-ray diffraction investigation of corneal structure in lumican-deficient mice. Investigative Ophthalmology and Visual Science 42, 1750-1756.

Quantock, A.J., Meek, K.M., Ridgway, A.E.A., Bron, A.J., Thonar, E.J.M.A., 1990. Macular corneal dystrophy - reduction in both corneal thickness and collagen interfibrillar spacing. Current Eye Research 9, 393-398.

Radner, W., Mallinger, R., 2002. Interlacing of collagen lamellae in the midstroma of the human cornea. Cornea 21, 598-601.

Radner, W., Zehetmayer, M., Aufreiter, R., Mallinger, R., 1998a. Interlacing and cross-angle distribution of collagen lamellae in the human cornea. Cornea 17, 537-543.

Radner, W., Zehetmayer, M., Skorpik, C., Mallinger, R., 1998b. Altered organization of collagen in the apex of keratoconus corneas. Ophthalmic Research 30, 327-332.

Rehany, U., Lahav, M., Shoshan, S., 1982. Collagenolytic activity in keratoconus. Annals of Ophthalmology 14, 751-754.

Sawaguchi, S., Fukuchi, T., Abe, H., Kaiya, T., Sugar, J., Yue, B.Y., 1998. Three dimensional electron microscopic study of keratoconus. Archives of Ophthalmology 116, 62-98.

Sawaguchi, S., Twining, S.S., Yue, B.Y., Wilson, P.M., Sugar, J., Chan, S.K., 1990. Alpha-1 proteinase inhibitor levels in keratoconus. Experimental Eye Research 50, 549-554.

Sawaguchi, S., Twining, S.S., Yue, B.Y., Chang, S.H., Zhou, X., Loushin, G., Sugar, J., Feder, R.S., 1994. Alpha-2-Macro-globulin levels in normal and keratoconus corneas. Investigative Ophthalmology and Visual Science 35, 4008-4014.

Sawaguchi, S., Yue, B.Y., Sugar, J., Gilboy, J.E., 1989. Lysosomal enzyme abnormalities in keratoconus. Archives of Ophthalmology 107, 15071510.

Sherwin, T., Brookes, N.H., Loh, I.P., Poole, C.A., Clover, G.M., 2002. Cellular incursion into Bowman's membrane in the peripheral cone of the keratoconic cornea. Experimental Eye Research 74, 473-482.

Smolek, M.K., McCarey, B.E., 1990. Interlamellar adhesive strength in human eyebank corneas. Investigative Ophthalmology and Visual Science 31, 1087-1095.

Somodi, S., Hahnel, C., Slowik, C., Richter, A., Weiss, D.G., Guthoff, R.F., 1996. Confocal in vivo microscopy and confocal laser-scanning fluorescence microscopy in keratoconus. German Journal of Ophthalmology 5, 518-525.

Teng, C.C., 1963. Electron microscope study of the pathology of keratoconus: part 1. American Journal of Ophthalmology 55, 18-47.

Wollensak, G., Sporl, E., Seiler, T., 2003. Behandlung von Keratokonus durch kollagenvernetzung. Ophthalmologe 100, 44-49.

Yue, B.Y., Sugar, J., Benveniste, K., 1984. Heterogenity in keratoconus: possible biochemical basis. Proceedings of the Society for Experimental Biology and Medicine 175, 336-341.

Zhou, L.L., Sawaguchi, S., Twining, S.S., Sugar, J., Feder, R.S., Yue, B.Y., 1998. Expression of degradative enzymes and protease inhibitors in corneas with keratoconus. Investigative Ophthalmology and Visual Science 39, 1117-1124. 